3. Kolsum U, Damera G, Pham TH, et al. Pulmonary inflammation in patients with chronic obstructive pulmonary disease with higher blood eosinophil counts. J Allergy Clin Immunol 2017;140(4):1181-1184.e7.

4. Pavord ID, Korn S, Howarth P, et al. Mepolizumab for severe eosinophilic asthma (DREAM): a multicentre, double-blind, placebo-controlled trial. Lancet 2012;380:651-9.

5. Bafadhel M. Eosinophils in COPD: are we nearly there yet? Lancet Respir Med 2017;5:913-4.

DOI: $10.1056 / N E J M c 1715454$

\title{
Diuretic Treatment in Heart Failure
}

TO THE EDITOR: The review article by Ellison and Felker (Nov. 16 issue) ${ }^{1}$ has a two-edged potential. Physicians are encouraged to use diuretics as first-line therapy for heart failure, but discontent with the drugs runs like a thread through the review. We had hoped that the section on other approaches and future directions would address how to minimize the use of diuretics. Long-term use of diuretic therapy for chronic heart failure may contribute to neurohumoral stimulation. ${ }^{2}$ Therefore, not only does skillful use of diuretic therapy remain fundamental to the successful management of heart failure, but so do strategies for their avoidance. First-line use of diuretics might influence adequate dosing of cardioprotective drugs, and the promotion of cardiac regeneration may become impractical. The latter is of particular importance the younger the patient with heart failure. ${ }^{3}$

Dietmar Schranz, M.D., Ph.D.

Pediatric Heart Center

Frankfurt, Germany

dietmar.schranz@kgu.de

No potential conflict of interest relevant to this letter was reported.

1. Ellison DH, Felker GM. Diuretic treatment in heart failure. N Engl J Med 2017;377:1964-75.

2. Schranz D, Voelkel NF. "Nihilism" of chronic heart failure therapy in children and why effective therapy is withheld. Eur J Pediatr 2016;175:445-55.

3. Mollova M, Bersell K, Walsh S, et al. Cardiomyocyte proliferation contributes to heart growth in young humans. Proc Natl Acad Sci U S A 2013;110:1446-51.

DOI: 10.1056/NEJMc1716477

TO THE EDITOR: In the sections on treatment of diuretic resistance and on adjuncts to diuretic therapy, the authors omit an important consideration. It has been reported that prolonged highdose treatment with furosemide can result in thiamine deficiency. ${ }^{1-3}$ Some studies have indicated that supplementation of thiamine in patients with heart failure improves cardiac function and urinary output. ${ }^{1,2,4}$ Administering thiamine to patients who receive continuing diuretic therapy is therefore in order. At least some patients may thus be spared the apparently paradoxical effect of "the more you give, the less you get."

Peter H. Berczeller, M.D.

New York University School of Medicine

New York, NY

peterberczeller@aol.com

No potential conflict of interest relevant to this letter was reported.

1. Katta N, Balla S, Alpert MA. Does long-term furosemide therapy cause thiamine deficiency in patients with heart failure? A focused review. Am J Med 2016;129(7):753.e7-753.e11.

2. Seligmann $\mathrm{H}$, Halkin $\mathrm{H}$, Rauchfleisch $\mathrm{S}$, et al. Thiamine deficiency in patients with congestive heart failure receiving longterm furosemide therapy: a pilot study. Am J Med 1991;91:151-5. 3. Misumida N, Umeda H, Iwase M. Shoshin beriberi induced by long-term administration of diuretics: a case report. Case Rep Cardiol 2014;2014:878915.

4. DiNicolantonio JJ, Niazi AK, Lavie CJ, O'Keefe JH, Ventura HO. Thiamine supplementation for the treatment of heart failure: a review of the literature. Congest Heart Fail 2013;19:214-22.

DOI: 10.1056/NEJMc1716477

TO THE EDITOR: Ellison and Felker report that the combination of hypertonic saline with high doses of loop diuretics has been proposed to improve renal dysfunction and natriuresis in congestive heart failure, although that approach has not been tested in robust trials. As reported by Gandhi et al., ${ }^{1}$ our group has conducted some trials on the role of high-dose furosemide and small-volume hypertonic saline solution infusion in the treatment of refractory congestive heart failure. The first, a randomized, single-blind study, ${ }^{2}$ showed that this treatment was associated with mortality reduction and clinical improvement during an extended follow-up period. A second study ${ }^{3}$ showed that patients who received hypertonic saline solution had a significantly faster reduction in brain natriuretic peptide levels and a lower incidence of readmission at follow-up than patients who did not receive it. A third study, ${ }^{4}$ which examined the effect of hypertonic saline solution on levels of natriuretic and immunoinflamma- 
tory markers after a short-term saline load, suggested a possible amelioration of myocardial stretching that is linked to heart failure. Thus, our findings showed efficacy of this therapy in patients with refractory congestive heart failure in improvement of quality of life, modulation of neurohormonal activation, and reduction of mortality and hospital readmissions.

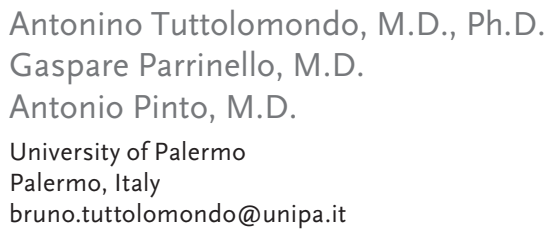

No potential conflict of interest relevant to this letter was reported.

1. Gandhi S, Mosleh W, Myers RB. Hypertonic saline with furosemide for the treatment of acute congestive heart failure: a systematic review and meta-analysis. Int J Cardiol 2014;173:139-45. 2. Licata G, Di Pasquale P, Parrinello G, et al. Effects of highdose furosemide and small-volume hypertonic saline solution infusion in comparison with a high dose of furosemide as bolus in refractory congestive heart failure: long-term effects. Am Heart J 2003;145:459-66.

3. Paterna S, Di Pasquale P, Parrinello G, et al. Changes in brain natriuretic peptide levels and bioelectrical impedance measurements after treatment with high-dose furosemide and hypertonic saline solution versus high-dose furosemide alone in refractory congestive heart failure: a double-blind study. J Am Coll Cardiol 2005;45:1997-2003.

4. Tuttolomondo A, Pinto A, Di Raimondo D, et al. Changes in natriuretic peptide and cytokine plasma levels in patients with heart failure, after treatment with high dose of furosemide plus hypertonic saline solution (HSS) and after a saline loading. Nutr Metab Cardiovasc Dis 2011;21:372-9.

DOI: 10.1056/NEJMc1716477

THE AUTHORS REPLY: Schranz suggests that diuretic use should be minimized in treating heart failure, owing to potential adverse neurohormonal effects. Although there is a dearth of data from randomized trials of the effects of diuretics on hard outcomes in chronic heart failure, small studies on diuretic discontinuation have been conducted. Grinstead et al. ${ }^{1}$ discontinued diuretics in 41 patients with stable heart failure with reduced ejection fraction, who were then randomly assigned to either lisinopril or placebo. A total of $71 \%$ required reintroduction of diuretics for congestive symptoms, regardless of concomitant treatment; baseline furosemide doses exceeding $40 \mathrm{mg}$ daily were predictive of diuretic reinitiation. More recently, 15 of 26 patients with previously stable heart failure $(58 \%)$ remained congestion-free for up to 1 year after diuretic withdrawal, although withdrawal was associated with a significant rise in plasma levels of atrial natriuretic peptide, even in those who did not have congestive symptoms. ${ }^{2}$ In those patients who remained congestion-free after diuretic withdrawal, plasma renin activity after 3 months was lower than at baseline, but the serum level of aldosterone and plasma levels of arginine vasopressin, endothelin-1, and norepinephrine remained unchanged.

Thus, although diuretic withdrawal poses substantial risk that congestive symptoms will recur, the condition of selected patients with stable heart failure may remain stable clinically. There are, however, no long-term intention-to-treat studies assessing the effects of this approach on morbidity and mortality among patients with heart failure. One should recall that the use of thiazide-like diuretics to treat hypertension substantially reduces the risk of heart failure, without increasing adverse outcomes, despite stimulation of the renin-angiotensin-aldosterone pathway. ${ }^{3}$

Berczeller makes the important point that thiamine deficiency has been reported to be a complication of high doses of furosemide and may worsen symptoms of heart failure. The fact that torsemide has not been reported to cause this adverse event may be another advantage of using that loop diuretic.

Tuttolomondo and colleagues expand on our mention of observations regarding hypertonic saline when used in combination with diuretics; they reference a meta-analysis by Gandhi and colleagues. ${ }^{4}$ We agree with the authors of that article that further study of such an approach with the use of adequate power, randomization, and blinding may be worthwhile.

David H. Ellison, M.D.

Oregon Health and Science University

Portland, OR

ellisond@ohsu.edu

G. Michael Felker, M.D.

Duke University

Durham, NC

Since publication of their article, the authors report no further potential conflict of interest.

1. Grinstead WC, Francis MJ, Marks GF, Tawa CB, Zoghbi WA, Young JB. Discontinuation of chronic diuretic therapy in stable congestive heart failure secondary to coronary artery disease or to idiopathic dilated cardiomyopathy. Am J Cardiol 1994;73: 881-6.

2. Galve E, Mallol A, Catalan R, et al. Clinical and neurohumoral consequences of diuretic withdrawal in patients with chronic, stabilized heart failure and systolic dysfunction. Eur J Heart Fail 2005;7:892-8. 
3. ALLHAT Officers and Coordinators for the ALLHAT Collaborative Research Group. Major outcomes in high-risk hypertensive patients randomized to angiotensin-converting enzyme inhibitor or calcium channel blocker vs diuretic: the Antihypertensive and Lipid-Lowering Treatment to Prevent Heart Attack Trial (ALLHAT). JAMA 2002;288:2981-97.
4. Gandhi S, Mosleh W, Myers RB. Hypertonic saline with furosemide for the treatment of acute congestive heart failure: a systematic review and meta-analysis. Int J Cardiol 2014;173: 139-45.

DOI: $10.1056 / N E J M c 1716477$

\section{Case 31-2017: A 19-Month-Old Girl with Failure to Thrive}

TO THE EDITOR: In the Case Record involving an emaciated 19-month-old girl with a ravenous appetite in whom the diencephalic syndrome was diagnosed, Curran et al. (Oct. 12 issue) ${ }^{1}$ do not discuss why a neuro-ophthalmological evaluation to rule out intracranial hypertension was not performed. The marked findings on imaging, which showed compromise of the optic chiasm, obstruction of the right foramen of Monro, and hydrocephalus, strongly suggest that the child's vision might have been affected and that nystagmus, papilledema, and neurodevelopmental delay might have been present. These signs and symptoms compel such clinical evaluations and were de rigueur before computed tomography and magnetic resonance imaging became available. This case underscores the worrisome trend in which technology is displacing simple clinical maneuvers that in this instance might have been more informative than many of the complex and expensive tests that were performed.

The underlying mechanisms by which this syndrome, in the presence of a high caloric intake, results in failure to thrive without impinging on linear growth are unknown. Such unexpected outcomes place current paradigms regarding energy balance into question. A better understanding of this rare syndrome may provide insights into more common conditions in which caloric intake is affected.

\section{Saul Malozowski, M.D., Ph.D.}

National Institute of Diabetes and Digestive and Kidney Diseases Bethesda, MD

sm007@nih.gov

The views expressed in this letter are those of the author and do not represent an official position of the Department of Health and Human Services.

No potential conflict of interest relevant to this letter was reported.

1. Case Records of the Massachusetts General Hospital (Case 31-2017). N Engl J Med 2017;377:1468-77.

DOI: $10.1056 /$ NEJMc1714806
TO THE EDITOR: Curran et al. describe "a 19-monthold girl with failure to thrive." Replacing the term "failure to thrive" with precise descriptive terminology, as defined in guidelines from the World Health Organization (WHO) ${ }^{1}$ and others, ${ }^{2,3}$ would help to facilitate the understanding of the prevalence, prognosis, and outcomes of malnutrition-associated conditions. Varying definitions of the imprecise historical term "failure to thrive" render comparisons across studies difficult, since as the case discussants note, there is "no universally agreed-on definition" for this term. This child's growth data supported a diagnosis of moderate acute malnutrition, which the WHO defines as a weight-for-height value of more than 2 SD below the median. ${ }^{1}$

Failing to appropriately define this child's nutritional status may have led to the strikingly large number of investigations that were conducted. Data that were published nearly 40 years ago indicate that laboratory investigations rarely identify a diagnosis that is not already suspected on the basis of the medical history and physical examination. ${ }^{4}$ In this rare but classic presentation of the diencephalic syndrome, an etiologic approach to defining malnutrition and the use of established terms to quantify it might have reduced the delay in diagnosis and the number of investigations.

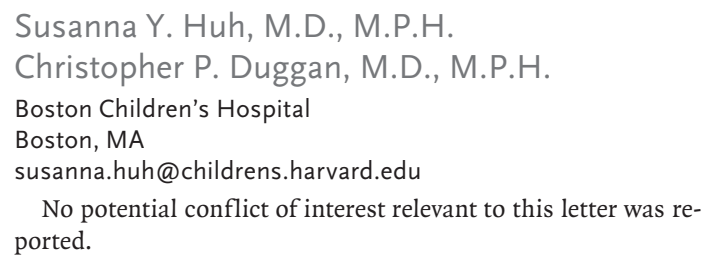
ported.

1. WHO child growth standards and the identification of severe acute malnutrition in infants and children: a joint statement by the World Health Organization and the United Nations Children's Fund. Geneva: World Health Organization, UNICEF, 2009.

2. Cederholm T, Barazzoni R, Austin P, et al. ESPEN guidelines on definitions and terminology of clinical nutrition. Clin Nutr 2017;36:49-64. 